\title{
Integración económica en América Latina: la visión teórica de la CEPAL confrontada con la evolución del proyecto en la región*
}

\section{Economic Integration in Latinamerica: The Theoretical Vision of ECLAC Against the Evolution Project in the Region}

\author{
Raúl Vázquez López ${ }^{1}$
}

Recibido: April 15, 2011

Aceptado: August 6, 2011

\section{Resumen}

Este artículo sintetiza las reflexiones relevantes que la CEPAL ha elaborado sobre la integración económica en América Latina, poniendo énfasis en el cambio de orientación ideológica de la Comisión hacia un sentido ortodoxo durante los años noventa. Al contrastar estas ideas con la evidencia empírica referente a la evolución del comercio intrarregional en los últimos años, resulta que los elementos que han dificultado históricamente el avance del proyecto siguen presentes, pero bajo formas más complejas relacionadas con las dinámicas actuales de la economía internacional. En particular, la conducción implícita de una integración parcial por parte de empresas transnacionales ha resultado en relaciones no equitativas entre los países y en un mayor aprovechamiento del proceso por parte de las economías más fuertes. El artículo finaliza entonces buscando revitalizar las reflexiones pioneras de la CEPAL y propone lineamientos para la construcción de un proyecto integracionista alternativo que sostenga la necesidad de rebasar los aspectos meramente comerciales de la integración.

Palabras claves: Integración, América Latina, dependencia, comercio, CEPAL.

\section{Summary}

This article tracks the main ECLAC ideas over the economic integration in Latin American underlying the change of the ideological background of ECLAC towards an orthodox sense during the nineties. When comparing those ideas with the evolution of intraregional trade in recent years, empirical evidence shows that elements that have historically hindered the projected progress are still present but in more complex forms related to the current dynamics of the international economy. In particular, the implicit management of a partial integration by transnational companies has resulted in unequal relationships between countries and in a wider use from the mentioned process by the strongest economies. The article ends, then, seeking to revitalize the pioneer ideas of ECLAC and proposes guidelines for the construction of an alternative integrationist project that supports the need of exceeding the purely commercial aspects of integration.

Keywords: Integration, Latin America, dependency, commerce, ECLAC, transnational companies.

* Un especial agradecimiento al proceso de dictamen anónimo de la revista cuyas valiosas observaciones permitieron mejorar el presente trabajo.

1. Doctor en Ciencia Política, Universidad del País Vasco. Investigador Titular del Instituto de Investigaciones Económicas (IIEc.), UNAM, México. <ravazz@hotmail.com>. 


\section{INTRODUCCIÓN}

En las últimas décadas, la integración latinoamericana se ha mantenido como una constante en el paradigma teórico de la Comisión Económica para América Latina (CEPAL) que por décadas tuviera una fuerte influencia sobre los modelos de desarrollo nacionales implementados en la región. Es particularmente notable la gran diversidad de contextos materiales e históricos en los cuales se han argumentado las ventajas de esta integración y los diversos perfiles ideológicos implícitos en su defensa. Por supuesto, la CEPAL, como todo organismo internacional institucionalizado, ha pretendido permanentemente presentar sus juicios como apegados a la realidad económica objetiva de cada etapa y sobre todo como independientes de marcos de análisis sesgados.

No obstante, el seguimiento histórico del tema desde una perspectiva estructural parece arrojar que, por lo menos en dos etapas diferenciadas, la propuesta ha sido presentada en aras de estrategias de desarrollo por demás distintas, incluso contradictorias. Como resultado, se han asociado toda una serie de objetivos a la integración, lo que ha generado la impresión de que el proceso regional pudiera ser la panacea para los problemas latinoamericanos y no una parte de un proyecto más general y sustantivo de desarrollo (Cuervo, 2000).

Ante el renovado interés por la integración en América Latina, en parte como resultado de los cambios en la conducción política de los gobiernos de algunos de sus países, es interesante contrastar un seguimiento de las diferentes reflexiones realizadas en el seno de la Comisión con la evidencia empírica recabada. En este marco, el presente artículo pretende reflexionar en torno a los aspectos económicos y comerciales de la integración latinoamericana destacando el aporte de las contribuciones pioneras de la CEPAL al tema.

Tras esbozar los elementos de la propuesta integracionista original de Raúl Prebisch y sus allegados, se aborda brevemente el rol de las empresas transnacionales (ETN) y de los Estados nacionales en el fracaso de la integración económica en América Latina. En la tercera parte, se pone en evidencia y se cuestiona el viraje ideológico de la CEPAL a partir de los años noventa, mientras que en el cuarto apartado se analiza la evolución reciente de los intercambios regionales desde la perspectiva de las bases de complementariedad productiva entre los países que supone. Finalmente, las conclusiones sintetizan algunos aspectos a considerar para la construcción de un proyecto alternativo.

\section{ELEMENTOS DE LA PROPUESTA ORIGINAL DE INTEGRACIÓN REGIONAL DE LA CEPAL}

Desde su origen, la propuesta de la integración latinoamericana causó grandes expectativas, quizás en gran medida porque su argumentación se insertó en la lógica de un cuerpo teórico de análisis y de un modelo de desarrollo coherente y mucho más general. Las ideas pioneras de Raúl Prebisch y allegados conformaban una estrategia que aunque esencialmente económica, era una visión multidisciplinaria que cuestionaba de forma integral los procesos de desarrollo en la región y sus modos históricos de inserción en el ámbito mundial. Se trataba en síntesis de una reflexión heterodoxa que emocionaba por la profundidad del análisis y por enunciar soluciones alternativas que prometían atacar de raíz los problemas económicos, políticos y sociales de América Latina.

En noviembre de 1956, el Comité de Comercio de la CEPAL solicitó a la Secretaria Ejecutiva de la Comisión la constitución de dos grupos de expertos, uno para el establecimiento gradual de un régimen de pagos multilaterales y otro para definir las características del mercado regional. En este contexto, se elaboraron una serie de recomendaciones que en las siguientes décadas se convertirían en las referencias teóricas del proyecto de integración económica (lo cual no deja de poner en evidencia tanto la riqueza de la conceptualización original como, en términos comparativos, la crisis teórica posterior). En la reflexión, destacan, por una parte, los argumentos de tipo teórico y por otra, los de carácter instrumental. 
El primer grupo de ideas se articula en torno a la visión según la cual los mercados nacionales eran reducidos para alcanzar condiciones de eficiencia. Para avanzar en dirección de una especialización productiva más sofisticada, de mayor contenido tecnológico, la estrategia de la industrialización por sustitución de importaciones (ISI), requería de la realización de economías de escala. Como resultado, el desarrollo de las industrias básicas, y particularmente de las productoras de bienes de capital (maquinaria) características de una etapa superior de especialización productiva en el modelo propuesto, necesitaba de la constitución de un mercado protegido más amplio (Prebisch, 1959).

La integración regional se asociaba entonces a la disponibilidad de un mercado común relativamente cautivo y de cierto tamaño, lógica que dicho sea de paso es muy similar a la que inspirara, en una primera instancia, la construcción del mercado europeo. A este argumento de peso a favor de la integración se agregaba en primer lugar la posibilidad de diversificar las importaciones y exportaciones, pero, en segundo término, de coordinar una política latinoamericana común frente a las dinámicas de la economía internacional.

La propuesta era también, en suma, un instrumento político que permitiría romper con una dependencia histórica en relación con los centros industriales. La subordinación de las estructuras productivas latinoamericanas a los requerimientos de las grandes empresas y de los mercados extranjeros se traducía en precios elevados de las importaciones y, en consecuencia, en altos costos del proceso sustitutivo. En palabras de Prebisch, la industrialización en la región se había desarrollado en el molde anacrónico de las relaciones comerciales del siglo XIX, caracterizadas por un escaso intercambio entre los países de América Latina.

Es importante recalcar que en la visión cepalina original, y hasta los años setenta, los autores de esta corriente entendían que la integración económica pasaba forzosamente por consensos políticos y que estos se reflejarían en la creación de mecanismos e instituciones comunitarios. A manera de ejemplo, Felipe Herrera escribía en una obra colectiva sobre el tema, compilada por Osvaldo Sunkel (1970), que: "La formación de un espacio económico [...] requiere de dos elementos esenciales: la voluntad política de los países de participar en un proceso de integración de sus economías y la existencia de instrumentos jurídicos e institucionales adecuados para traducir las decisiones políticas en programas concretos de acción oportuna y coordinadamente ejecutados" (311).

En lo referente a la instrumentación del mercado común, se propuso como motor del proyecto, y a la luz de la ineficiencia relativa de la mayoría de las empresas nacionales sobreprotegidas por las distintas barreras a la entrada de productos de importación, la creación de nuevas industrias que absorbieran el incremento poblacional. Otro aspecto esencial era el ritmo gradual y progresivo al cual debería aunarse tanto la eliminación de derechos aduaneros como la transformación de la planta productiva existente.

Finalmente, según lo afirmaba Presbich (1959), se sugerían principios de solidaridad para con las naciones de menor desarrollo al interior del bloque, tales como: “...un promedio más alto de derechos que el que se acordara como meta para los países industrialmente más avanzados; y por otro las concesiones especiales que éstos otorguen para abrir sus mercados a exportaciones de manufacturas de aquellos países de incipiente industrialización" (30).

El objetivo era incrementar los intercambios de productos manufactureros entre los países de la región como base para la construcción de un comercio más especializado, justo y de mayor contenido en tecnología. En el marco de estrategias nacionales de desarrollo de largo plazo concentradas en la industrialización, se buscaba entonces construir patrones de especialización productiva con mayores grados de complementariedad entre sí. No obstante, en un principio se planteaba evitar recrear un esquema de división regional del trabajo no equitativo como el existente en el ámbito global caracterizado por la segmentación entre naciones productoras de bienes primarios y centros industriales. Al sintetizar los conceptos fundamentales en los que se apoyaba la idea de la integración, Tavares y Gomes (1998) señalan 
la oposición centro/periferia, la restricción externa y la escasez de capital y tecnología.

\section{ESTADOS NACIONALES Y EMPRESAS TRANSNACIONALES EN EL FRACASO LATINOAMERICANO}

En la práctica, estos primeros postulados integracionistas de la CEPAL nunca fueron implementados, así lo muestra el limitado contenido y el posterior fracaso de las primeras experiencias de integración económica y comercial en la región, tales como la Asociación Latinoamericana de Libre Comercio (ALALC), el Mercado Común Centroamericano (MCC), la Comunidad del Caribe (CARICOM) y el Pacto Andino. Los instrumentos empleados por lo general fueron un arancel externo común, la rebaja de los aranceles entre los países de la región, la armonización de las normas y procedimientos administrativos y el desarrollo de una infraestructura de transporte y comunicaciones.

Un elemento fundamental en la imposibilidad de avanzar, según los preceptos teóricos propuestos, fue la incapacidad o falta de voluntad de los gobiernos nacionales e instituciones internacionales competentes para revertir las tendencias de la inserción de las economías latinoamericanas en el ámbito global. Si bien el crecimiento económico registrado en América Latina en los años 60 y 70 permitió ampliar los mercados nacionales, sus rasgos íntimamente relacionados con su carácter dependiente agravaron diversas particularidades estructurales propias del atraso del aparato de fabricación de la región. De hecho, las estrategias globales de búsqueda de mercados y factores productivos, expansión y localización de las ETN no eran compatibles con la lógica de complementación de los patrones de especialización productiva propia de un proyecto integral y sostenible de integración.

Desde esta perspectiva, el control del proceso de crecimiento económico, y en cierta medida el de la estrategia ISI en su conjunto, lo tuvieron principalmente las ETN. Estas empresas fomentaron cierto tipo de regionalización y hasta de complementación, pero sólo en la medida en la que el proceso les permitió profundizar en la "transnacionalización" a través de pautas delineadas por las casas matrices (Fajnzylber, 1970). En síntesis, los esquemas de regionalización permitieron a las ETN avanzar en la articulación de sus subsidiarias en los diferentes países, pero al adoptar las formas necesarias a este fin, bloquearon en América Latina procesos de aprendizaje y de construcción de capacidades tecnológicas (Sunkel, 1998).

Se debe destacar que estas transformaciones se realizaron particularmente en muchos casos en los países de mayor desarrollo industrial con la colaboración formal de los Estados nacionales. Estos últimos implementaron diversos mecanismos para atraer a la inversión extranjera a la par que mantuvieron elevadas barreras en contra de las importaciones a sus mercados, estrategia que benefició por partida doble a los negocios de las ETN e impidió la conformación de un bloque regional según los principios de la propuesta cepalina original.

El colofón del modelo establecido, que Cardoso y Faletto (1970) calificaran de "desarrollo dependiente y asociado", se dio entonces con la crisis de la deuda en los años ochenta y el consiguiente cambio de orientación ideológica, cuya difusión se hizo desde los organismos internacionales, entre los que se encontró a una CEPAL teóricamente "revisada". En el periodo 1982-1990, las transferencias netas de la región al exterior, fueron de alrededor de 220 mil millones de dólares, cifra superior al 7\% del PIB latinoamericano en dólares de 1980 (CEPAL, 1991).

Bajo la presión de una situación económica regional muy deteriorada, las soluciones planteadas partieron entonces de la evidencia del fracaso del modelo de la ISI desde un enfoque sesgado que atribuía a los gobiernos nacionales la total responsabilidad de los resultados obtenidos, pero que ignoró el rol que las ETN y las diversas formas de dependencia habían significado. Mediante reformas estructurales se buscó, entre otros objetivos, abrir de manera completa y definitiva las economías, hasta entonces parcialmente protegidas, a las inversiones e importaciones provenientes de 
las naciones más avanzadas. El esquema de apertura indiscriminada y desregulación significó además la culminación del proceso del supuesto reemplazo del Estado por la empresa privada en el rol de liderazgo del modelo de desarrollo económico.

\section{EL CAMBIO DE ORIENTACIÓN IDEOLÓGICA EN LA CEPAL}

En este contexto y a partir de los años noventa, la CEPAL retoma la propuesta integracionista desde un enfoque que en su esencia es diametralmente opuesto al original. En la argumentación de la Comisión a favor de la integración latinoamericana se mantienen la mayoría de los elementos generales "clásicos" de la visión estructural, pero en esta ocasión son vistos como un medio de profundización del proceso de apertura y desregulación indiscriminadas de las economías de la región.

Los autores agrupados en torno a la CEPAL postulan que con la superación de la ISI, las posibilidades de la integración se ven reforzadas en lo referente a su capacidad para permitir el acceso a economías de escala, progreso técnico, incrementos en los niveles de productividad e inversión así como para contribuir con un cambio estructural en dirección de mercados más competitivos. Se supone que la globalización genera áreas de oportunidad para el crecimiento industrial, menores costos de cara a la integración y un contexto de mayor eficiencia para las empresas (Rosenthal, 1999).

Palma (1999), haciendo referencia a la Comisión, habla incluso de un consenso latinoamericano básico en cuanto a los requisitos mínimos de una política de desarrollo productivo, entre los que menciona tanto la apertura comercial como la mayor influencia de las fuerzas del mercado y el sector privado para liderar el modelo de desarrollo. Y va más lejos aún al caer en una concepción teórica lineal de la evolución económica de las naciones y otorgar a dichos elementos el grado de "prerrequisitos" de la integración para casi todos los países de la región.
Con esta "nueva orientación", la CEPAL deposita su confianza en las reformas estructurales y propone un regionalismo extensivo a nivel planetario cuyo fin último es una liberalización comercial mundial. La contradicción, aunque aparentemente abordada en los diferentes documentos elaborados por la CEPAL, tales como un regionalismo abierto en América Latina y el Caribe y la integración económica al servicio de la transformación productiva con equidad (1994a) resulta, por lógica, flagrante. ¿Qué sentido tiene, entonces, el regionalismo? ¿En qué se distingue fundamentalmente de un proceso de apertura comercial indiscriminada?

A manera de ejemplo, el resumen del documento antes citado asume una clara postura en relación con la apertura del comercio de los servicios, área de particular atraso en la región:

Dado que los procesos de privatización y de desreglamentación de los servicios pueden ampliar el número de empresas en condiciones de suministrarlos, a la vez que abren la posibilidad de aprovechar mejor la cercanía geográfica y la afinidad cultural de los países de la región, se fortalecerían las condiciones favorables a un incremento del comercio de servicios y de la inversión de origen intra y extraregional en este sector en particular." (1994b, 8).

Diversos aspectos son de destacar en estas líneas. Primeramente, la orientación de corte ortodoxo favorable a los procesos de privatización y desreglamentación que constituye una autentica revisión y renuncia a la esencia teórica del estructuralismo original. En segunda instancia, la importancia que la CEPAL otorga al sector servicios, y en concreto al financiero, para el éxito del mercado regional en el contexto de la insuficiencia de capital en América Latina. Es, sin embargo, una interpretación exenta de toda concepción "dependentista" y en la que la entrada de capital extranjero es parte de las "nuevas" condiciones económicas caracterizadas como de oportunidad.

Un tercer aspecto a subrayar es, en este orden de ideas, la aparente neutralidad en lo referente al origen (intra o extraregional) tanto de la inversión, como en 
otras partes del documento, de los acuerdos comerciales (1994b). Sobre el último punto, el documento también elimina las consideraciones sobre un tema hasta entonces vital en las reflexiones estructuralistas: el asunto de la propiedad del aparato productivo. Ya que en la mayoría de los países de la región las grandes corporaciones extranjeras reinvierten, en el mejor de los casos, una parte reducida del beneficio que realizan, todo indica que el origen de los capitales es particularmente relevante en un debate ineludible en torno a la regulación estatal de la inversión extranjera y de la propiedad de las industrias en sectores estratégicos.

Por citar un ejemplo, en el Perú se estima que el Estado dejó de recaudar del sector minero alrededor de 2,700 millones de dólares entre 2006 y 2007, al no cobrar las regalías ni aplicar el impuesto a las ganancias extraordinarias a las principales corporaciones que contaban con contratos de estabilidad tributaria (Padilla, 2009). Se debe mencionar que en 2006 , las mayores empresas del sector obtuvieron alrededor de 3,448 millones de dólares de ganancias adicionales y en 2007 acumularon otros 4,137 millones (Salazar, 2008).

En este orden de ideas, el argumento según el cual las políticas de apertura han tenido fuertes beneficios para las economías de tamaño intermedio en la región, entre ellas las de Chile, Perú y Colombia, parece obviar la profundidad y complejidad de los mecanismos de las relaciones económicas implícitas en el contexto reciente de la globalización. En primera instancia, estas políticas en la mayoría de las naciones latinoamericanas datan de los años ochenta y sus resultados deben ser evaluados conforme a un periodo de tiempo acorde y no limitado a una evolución coyuntural de tres o cuatro años. Al respecto, el déficit en cuenta corriente acumulado por estos tres países de 1980 al 2009 es de 116,559.1 millones de dólares, mientras que el dato para el periodo 1994-2009 es negativo en 68,951.9 millones ${ }^{1}$.

Un segundo aspecto a considerar es que los crecientes ingresos obtenidos por algunas naciones latinoamericanas mediante las exportaciones de commmodities y

1 Calculado a partir de CEPAL (2011). productos primarios, principalmente a China, es un fenómeno muy reciente que pudiera ser coyuntural y que tiene por origen el fuerte incremento de los precios internacionales de estos bienes. Este incremento, suscitado entre otras cosas por las tasas de crecimiento de la economía de dicho país asiático, no es entonces una consecuencia directa de las políticas de apertura cuyo fracaso evidencian los déficit acumulados en los saldos en cuenta corriente antes mencionados, y que para América Latina en su conjunto equivalen a 367, 857.9 millones de dólares en el lapso 1994-2009².

Sin embargo, el punto nodal de la reflexión que pone en tela de juicio los beneficios de la política de apertura y la integración comercial regional, tal y como se ha venido gestando, tiene que ver con las dinámicas de la economía internacional actual, su conducción por parte de las ETN y sus implicaciones para el desarrollo latinoamericano. A manera de primera ilustración y para terminar con el ejemplo de Chile, Colombia y Perú, economías con características sumamente distintas que aquí hemos reagrupado de forma arbitraria por su pretendido tamaño "medio", el considerable incremento en los precios de los productos que exportan y sus crecientes ingresos por ventas al exterior en los últimos años dependientes de los movimientos de mercados globales altamente especulativos, los ha vuelto muy vulnerables a las condiciones internacionales. En 2008, el déficit en cuenta corriente de los tres países sumó 14,476.4 millones de dólares, cifra que supera los saldos positivos obtenidos en conjunto en el periodo 2004-2007 $(11,518.5 \text { millones })^{3}$.

En el caso peruano, la conducción implícita de la apertura recae en menor medida en ETN y determinados sectores, particularmente en el rubro de industrias productoras o procesadoras de recursos naturales que se han visto beneficiadas al aumentar sus ventas tanto a China como al interior de la región. No obstante, las exportaciones no tradicionales han venido perdiendo peso en términos relativos en la canasta de ventas al extranjero por el alza de ciertos precios. Aún así, los

2 Calculado a partir de CEPAL (2011).

3 Calculado a partir de CEPAL (2011). 
datos permiten ejemplificar cómo los mecanismos que las casas matrices de ETN ejercen en el proceso no sólo determinan el valor y sentido de los flujos comerciales intrarregionales en América Latina, como se analizará más adelante, sino que también impiden que los mayores ingresos por exportaciones tengan derramas positivas sobre el resto del aparato productivo.

Al comparar las estadísticas del comercio de bienes en el año 2000 con respecto del 2009, se ve que las exportaciones del Perú registraron un fuerte incremento de $286.6 \%$, el valor unitario de las ventas al exterior creció en $105.4 \%$, mientras que el índice de términos del intercambio lo hizo en $29.1 \%$. No obstante, en dicho lapso, resultado del mayor dinamismo externo, los intereses pagados a la inversión extranjera aumentaron en $268 \%$ alcanzando un monto de 8,251.9 millones de dólares en 2009 , es decir $30.7 \%$ de los ingresos totales por exportaciones de bienes. Como colofón de este funcionamiento, el saldo en cuenta corriente del país significó una salida neta de divisas por 3,881.9 millones de dólares en el periodo 2000-2009 de supuesta bonanza financiera ${ }^{4}$.

De hecho, en el ámbito latinoamericano, en los últimos años, la acumulación de resultados económicos y sociales negativos producto de las medidas de reforma estructural aplicadas en América Latina, y el rechazo generalizado al cuerpo teórico que inspirara el llamado consenso de Washington, han hecho renacer entre otros aspectos, la voluntad política de los Estados nacionales de la región en torno al proyecto integracionista latinoamericano sobre otras bases. En consecuencia, la CEPAL parece estar "redescubriendo" parte de las virtudes olvidadas de las reflexiones originales de Prebisch (por supuesto desde un enfoque mucho más moderado que pudiera calificarse de neo-schumpeteriano). En palabras de Alicia Bárcena (2009), Secretaria Ejecutiva de la CEPAL, la integración latinoamericana se mantiene hoy en día como uno de los ejes de la estrategia de la institución para fortalecer las capacidades de competitividad e innovación de la región, a partir de: "facilitar el intercambio de experiencia entre los países y fortalecer

4 Calculado a partir de CEPAL (2011). la coordinación y cooperación regional para la búsqueda de nichos de integración y complementariedad entre los países de la región" (11).

\section{LA EVIDENCIA EMPÍRICA: COMERCIO INTRARREGIONAL Y COMPLEMENTARIEDAD}

En términos generales, la evolución reciente (19952009) del comercio en la región, no traduce un avance significativo de las relaciones económicas entre los países. En el marco de un acelerado crecimiento de los intercambios mundiales en los últimos años que, como referencia permitió a los miembros de la Asociación Latinoamericana de Integración (ALADI) triplicar el valor de sus ventas al exterior, la participación de las exportaciones al interior del bloque con respecto al total incluso se redujo, pasando de $17.2 \%$ en 1995 a $14.7 \%$ en el 2009 ( $\sin$ contar a Cuba) (ver Tablas 1 y 2).

En su conjunto, la región ha mejorado su posición comercial en el mercado de bienes en relación con el resto del mundo, fundamentalmente debido al superávit que las economías sudamericanas más fuertes han conseguido tras su creciente integración a la dinámica global. El saldo positivo en este rubro de Argentina, Brasil, Chile y Venezuela sumó en 2009 casi 70 mil millones de dólares (ver Tabla 1). Con excepción de Venezuela, cuya economía es altamente dependiente del petróleo, y a pesar de contar con patrones de especialización productiva muy distintos, estos países tienen en común el haber experimentado incrementos en sus grados de apertura comercial. En Argentina, el comercio exterior significó 31.1\% del PIB en 2009 cuando en 1995 este dato era del 15.9\%. Por su lado, en Brasil, esta participación aumentó del 13\% al 17.8\%,y en Chile del $42.6 \%$ se incrementó a $58.9 \%$.

No obstante, es Brasil, con el menor grado de apertura de las naciones consideradas pero con una economía

5 Calculado como el peso de la sumatoria de las exportaciones y las importaciones en el PIB de cada país, a partir de datos de la CEPAL (2011) y la ONU (2011). 
diversificada y un mercado interno en crecimiento, el país con el más alto superávit y el que ha mejorado en mayor medida su posición de intercambio de bienes con el mundo. En contraposición, México, cuyo comercio exterior representó en 2009 el 53\% de su PIB, con una planta de fabricación muy concentrada en sectores exportadores de productos con alto contenido tecnológico pero dominados por subsidiarias de ETN como el automotriz o el electrónico, es la nación que registra en ese mismo año el mayor déficit $(-4,672.53)$ (ver Tabla 1).

Tabla 1.

Exportaciones Totales de Países Latinoamericanos Seleccionados, 1995-2009 (Millones de dólares y porcentajes)

\begin{tabular}{lrrrrr}
\hline \multirow{2}{*}{ Países } & \multicolumn{2}{c}{ Exportaciones } & Tasa de & \multicolumn{2}{c}{ Saldo en Balanza Comercial } \\
\cline { 2 - 3 } \cline { 5 - 6 } & \multicolumn{1}{c}{1995} & 2009 & Crecimiento & 1995 & \multicolumn{1}{c}{2009} \\
\hline Argentina & $20,962.6$ & $55,669.1$ & 165.6 & 840.93 & $15,375.68$ \\
Bolivia & $1,181.2$ & $5,296.7$ & 348.4 & -215.04 & 887.76 \\
Brasil & $46,504.9$ & $152,995.0$ & 229.0 & $-7,229.35$ & $25,347.41$ \\
Chile & $15,901.1$ & $53,731.7$ & 237.9 & 998.09 & $11,304.33$ \\
Colombia & $10,201.0$ & $32,853.0$ & 222.1 & $-3,682.44$ & -44.69 \\
Ecuador & 4361.5 & 13724.3 & 214.7 & 166.35 & $-1,368.97$ \\
México & $79,540.7$ & $229,712.3$ & 188.8 & $7,087.67$ & $-4,672.19$ \\
Paraguay & 919.3 & $3,167.0$ & 244.5 & $-2,216.54$ & $-3,772.82$ \\
Perú & $5,439.7$ & $26,738.3$ & 391.5 & $-2,144.34$ & $4,868.52$ \\
Uruguay & $2,106.2$ & $5,385.5$ & 155.7 & -759.57 & $-1,521.22$ \\
Venezuela & $19,093.0$ & $56,583.1$ & 196.4 & $8,301.76$ & $17,906.46$ \\
\hline Total & 206211.3 & 635855.7 & 208.4 & $1,147.52$ & $64,310.20$ \\
\hline
\end{tabular}

Fuente: Elaboración propia con datos de la ONU (2011).

Se debe señalar también que en el periodo considerado, los dos países de la agrupación que tuvieron las más altas tasas de crecimiento de sus exportaciones son del área andina: Perú (391.5\%) y Bolivia (348.4\%), y cuyo crecimiento se caracteriza por haber apuntalado este proceso en una creciente dependencia con relación a productos primarios ligados a la extracción de minerales y derivados del petróleo. De esta forma, el gas natural representó 36.3\% de los envíos de Bolivia al extranjero en el 2009, mientras que en 1995 su principal producto de exportación eran el zinc y sus concentrados, que sumaban el $12.8 \%$ del total de sus exportaciones ${ }^{6}$.
El desempeño comercial de las distintas economías de la región en la dinámica mundial está entonces actualmente condicionado tanto por las dotaciones en recursos naturales de cada país como por el tamaño, potencial y grado de aprovechamiento de los respectivos mercados internos. Al respecto, como reflejo del predominio cada vez más visible de algunas naciones en América Latina, el espacio regional ha sido utilizado como una extensión de determinados mercados nacionales. Como dato, en 2009, las exportaciones intrarregionales de Brasil y Argentina en la ALADI representaron 56\% del total ( sin contar a Cuba) (ver Tabla 2).

6 Calculado a partir de datos de la CEPAL (2011). 
Tabla 2.

Exportaciones Intrarregionales entre Países Latinoamericanos Seleccionados, 1995-2009

(Millones de dólares y porcentajes)

\begin{tabular}{lrrrrr}
\hline \multirow{2}{*}{ Países } & \multicolumn{2}{c}{ Exportaciones } & Tasa de & \multicolumn{2}{c}{ Saldo en Balanza Comercial } \\
\cline { 2 - 3 } \cline { 5 - 6 } Argentina & \multicolumn{1}{c}{1995} & 2009 & Crecimiento & 1995 & 2009 \\
\hline Bolivia & $9,624.6$ & $22,887.1$ & 137.8 & $3,764.8$ & $7,638.8$ \\
Brasil & 422.1 & $3,103.5$ & 635.3 & -110.6 & 592.2 \\
Chile & $9,974.1$ & $29,619.5$ & 197.0 & -629.8 & $7,788.7$ \\
Colombia & $2,984.3$ & $8,182.8$ & 174.2 & $-1,051.0$ & $-4,164.9$ \\
Ecuador & $2,392.5$ & $8,042.2$ & 236.1 & -890.6 & -228.7 \\
México & 761.3 & $3,264.7$ & 328.8 & -486.7 & $-1,417.1$ \\
Paraguay & $2,853.0$ & $9,828.4$ & 244.5 & $1,438.9$ & $1,708.4$ \\
Perú & 596.3 & $2,195.8$ & 268.2 & $-1,470.6$ & $-1,180.2$ \\
Uruguay & 888.6 & $3,762.2$ & 323.4 & $-1,641.4$ & $-3,309.1$ \\
Venezuela & $3,115.0$ & $2,026.0$ & 81.7 & -354.1 & $-1,857.3$ \\
\hline Total & $35,552.7$ & $93,780.0$ & 163.8 & $-1,365.0$ & $-12,047.9$ \\
\hline
\end{tabular}

Fuente: Elaboración propia con datos de la ONU (2011).

Estos desequilibrios se traducen en diferentes niveles y tipos de aprovechamiento de los intercambios latinoamericanos. Las llamadas "fuerzas del mercado" y el "regionalismo abierto", que últimamente han dictado en gran medida las pautas de esta evolución comercial, parecen encauzar a los países en dirección de patrones exportadores internacionales basados en sus dotaciones factoriales y no en función de esquemas de integración regional complementarios, lo que explica que en las economías pequeñas no se hayan podido desarrollar ventajas competitivas dinámicas.

En consecuencia, las diferencias existentes entre las estructuras productivas de las naciones de América Latina han obstaculizado el avance de la integración y se han traducido en la materialización de ganadores y perdedores de un proceso parcial ${ }^{7}$. En 2009 en el marco de la ALADI, solo Argentina y Brasil registraron un superávit considerable en sus intercambios intrarregionales de productos, lo que se tradujo en saldos negativos para

Para el caso del Mercado Común del Sur (MERCOSUR) ver Ríos, 2003. el resto de los países de la Asociación, con la excepción de Bolivia y México (ver Tabla 2).

En síntesis y a grandes rasgos, mientras las economías de menor tamaño venden esencialmente materias primas e insumos a los países "lideres" de la región, estos últimos han utilizado los acuerdos comerciales tanto para consolidar sus eslabonamientos de fabricación, cuando les es posible, como para vender sus excedentes productivos realizando economías de escala en sus procesos productivos. Argentina y Brasil en particular, puesto que México se encuentra en la órbita de los Estados Unidos, se han beneficiado del mercado regional como plataforma de expansión de sus exportaciones a terceros países y de creación de ventajas comparativas dinámicas ${ }^{8}$.

Una de las principales explicaciones de la dinámica desigual de los intercambios de mercancías al interior de la región está ligada al control de los flujos comerciales

8 En lo referente a Argentina y Brasil, ver Bekerman \& Rikap, 2010 . 
por parte de las grandes empresas transnacionales. En el periodo 1995-2009, el comercio intrarregional por grandes categorías económicas se concentró en los bienes de capital y en los equipos de transporte y obedeció en muchos casos a las estrategias de localización de las filiales de las ETN. Dicho funcionamiento es concomitante con la progresiva concentración y especialización de los aparatos productivos nacionales, producto de la reestructuración que han experimentado a raíz de la apertura. A manera de ejemplo de este proceso, la quiebra de gran parte de la industria metalmecánica en varios países latinoamericanos resultó en la mayoría de los casos en el establecimiento de ETN de origen extranjero que monopolizaron los mercados locales con productos relacionados utilizándolos como plataformas de exportación en la región.

Un detalle del deterioro ocasionado en este rubro por la apertura y la consiguiente inserción de estas industrias en cadenas globales de valor es el de la destrucción del régimen tecnológico asociado a la producción de los automóviles Ford en Argentina, documentado por Cimoli y Katz (2002). En su trabajo, ambos autores señalan cómo la subsidiaria de la transnacional en el país sudamericano se transformó de una empresa que adaptaba los modelos extranjeros a las necesidades del mercado doméstico con un $90 \%$ de contenido local, empleando mano de obra calificada e invirtiendo en investigación y desarrollo en una planta ensambladora de componentes importados sin ningún efecto positivo sobre el desarrollo de las capacidades tecnológicas nacionales y pobremente integrada con la industria metalmecánica del país.
La evolución observada debe ser interpretada entonces como la continuidad del proceso de expansión corporativa de las ETN en América Latina a partir de estrategias globales y en el cual, los mecanismos de regionalización siguen siendo aprovechados para consolidar los eslabonamientos internacionales de valor de cada firma. Al no existir un contrapeso a dicha tendencia bajo la forma de proyectos nacionales que articulen de forma consecuente los objetivos integracionistas, la distribución de los beneficios de los acuerdos es desigual no sólo entre países sino al interior de los mismos, beneficiando principalmente al capital extranjero.

Otro ejemplo en este sentido es el rezago en el comercio de servicios en América Latina. En 2009, las importaciones en este rubro superaron en más de 34 mil millones de dólares a las exportaciones, cifra superior en $132.2 \%$ a la registrada en 1995 (ver Tabla 3). Si se considera que en la era de la información los servicios exportables se caracterizan por elevados niveles tanto de valor agregado como de contenido implícito en conocimiento, el creciente déficit está relacionado con la desfavorable evolución de los precios para la región de ciertas actividades del sector terciario, muchas de ellas anteriormente parte del proceso productivo, es decir con un deterioro generalizado de los términos del intercambio.

En este orden de ideas, la tendencia a la baja de la participación de los servicios en las ventas al exterior de los países latinoamericanos se traduce en un empobrecimiento de los perfiles exportadores y de especialización de dichas naciones. No obstante,

Tabla 3.

Comercio de Servicios en América Latina, 1995-2009 (Millones de dólares y porcentajes)

\begin{tabular}{crrccr}
\hline Años & 1995 & 2009 & $\begin{array}{c}\text { Tasa de } \\
\text { Crecimiento }\end{array}$ & $1995-2002$ & 2002-2009 \\
\hline Exportaciones & $36,746.1$ & $94,878.0$ & 158.2 & $365,085.7$ & $621,168.1$ \\
Importaciones & $51,413.2$ & $128,935.1$ & 150.8 & $494,935.9$ & $772,030.1$ \\
Saldo & $-14,667.07$ & $-34,057.05$ & -132.2 & $-129,850.2$ & $-150,862.0$ \\
\hline
\end{tabular}

Fuente: Elaboración propia con datos de CEPAL (2011). 
es también el resultado de la sobre-valuación que realizan las casas matrices de las ETN de sus funciones, principalmente mediante el establecimiento de precios de transferencia desiguales al interior de los grupos corporativos que gobiernan, y que les permite apropiarse de los beneficios resultantes de la actividad productiva global.

Todo parece, entonces, indicar que la ventaja competitiva en materia de servicios por parte de los países desarrollados entraña nuevas formas de dependencia para las naciones latinoamericanas y que estas formas se encuentran particularmente asociadas a las necesidades de funcionamiento de los grandes grupos transnacionales en el ámbito global. Resulta, en consecuencia, evidente que las reformas estructurales y los procesos de privatización implementados a partir de los años ochenta en la región fomentaron la monopolización de los mercados locales y, contrariamente a lo que sostuviera la CEPAL todavía en los años noventa, no sentaron tampoco las bases para el crecimiento del comercio intrarregional de productos del sector terciario.

\section{CONCLUSIONES}

La visión cepalina original de la integración latinoamericana se inserta en una estrategia multidisciplinaria e integral tendiente a sacar a las naciones de la región del subdesarrollo y a superar su condición dependiente y periférica. Al respecto, la revisión realizada por la propia CEPAL a partir de los años noventa de estos postulados pioneros, que nunca fueron comprobados empíricamente, debe ser considerada co-responsable de una reorientación teórica cuya implementación erosionó los tejidos productivos y bloqueó procesos de aprendizaje en las distintas economías.

Desde los inicios de la implementación de los proyectos de industrialización por sustitución de importaciones en América Latina y de acuerdo con Sunkel (1998), distintos grupos de las oligarquías locales han desarrollado "vínculos estrechos y sistemáticos con instituciones financieras internacionales y con gobiernos de países desarrollados" (236). Esto ha imposibilitado permanentemente a los Estados nacionales para fungir como contrapeso de las disposiciones de las ETN en aras de una política económica de origen más democrático.

Como resultado, la lógica de la división internacional del trabajo, capitaneada por los intereses de las ETN, ha dictado las pautas y formas de un proceso parcial de integración en América Latina. En un contexto de liberalización casi forzada de los intercambios para los países subdesarrollados, la evolución reciente de las relaciones comerciales al interior de la región refleja la continuidad y profundización de la dependencia de los diferentes modelos nacionales de desarrollo con respecto de una jerarquía mundial de centros de control y decisión política y económica, pero bajo esquemas más complejos.

Otro tipo de integración es, sin embargo, posible, en gran parte debido a los elementos estructurales y normativos de un proyecto alternativo que se encuentran en las reflexiones pioneras de la CEPAL. Entre estas tenemos, por ejemplo, el establecimiento de regulaciones que armonicen las asimetrías estructurales, de exigencias institucionales a las inversiones multinacionales y la coordinación de regímenes de reconversión industrial y de comercio exterior a partir de la formación de instituciones multinacionales autónomas (particularmente de los intereses de las ETN) y dotadas de las suficientes atribuciones y flexibilidad operacional. Estos cambios, entre otros, pudieran convertirse en un primer paso hacia un proceso más equitativo y ventajoso para América Latina.

En este sentido es indudable que, en el marco actual, una integración latinoamericana capaz de hacer avanzar al conjunto de sus miembros hacia una mayor independencia en relación con los intereses de los grandes corporativos transnacionales no puede resultar de un proceso acotado a la liberalización del comercio intrarregional. El prerrequisito fundamental e indispensable es entonces una profunda democratización de los estados nacionales que solo puede hoy en día resultar de una movilización consciente y organizada de la sociedad civil. 


\section{Referencias}

Bárcena, A. (2009, septiembre). Competitividad: visión de organismos regionales. Foro de Competitividad de las Américas III, Santiago de Chile.

Bekerman, M., \& Rikap, C. (2010). Integración regional y diversificación de exportaciones en el Mercosur: el caso de Argentina y Brasil. Revista CEPAL, 100, 169-191.

Cardoso, F. H., \& Faletto, E. (1970). Dependencia y desarrollo en América Latina. México, D.F.: Siglo Veintiuno Editores.

Cimoli, M., \& Katz, J. (2002). Structural Reforms, Technological Gaps and Economic Development. A Latin American Perspective. Serie Desarrollo Productivo, 129. Santiago de Chile: CEPAL.

Comisión Económica para América Latina y el Caribe (CEPAL) (2011). Anuario estadístico de América Latina y el Caribe, 2010. Santiago de Chile: CEPAL.

(1994a). El regionalismo abierto en América Latina y el Caribe: la integración económica al servicio de la transformación productiva con equidad. Santiago de Chile: CEPAL.

(1994b). El regionalismo abierto en América Latina y el Caribe: la integración económica al servicio de la transformación productiva con equidad. Síntesis, en línea, <http:/www.eclac.org/publicaciones/ $\mathrm{xml} / 7 / 4377 / \operatorname{lcg} 1801 \mathrm{e} . \mathrm{htm}>$ (Recuperado el 2 de diciembre, 2010).

(1991). La transferencia de recursos externos de América Latina en la posguerra. Serie Cuadernos de la CEPAL, 67. Santiago de Chile: Naciones Unidas.

Cuervo, M. (2000). El sistema de integración económica y la importancia de los efectos estáticos. Análisis Económico, XV(32), 111-130.

Fajnzylber, F. (1970). Estrategia industrial y empresas transnacionales: posición relativa de América Latina y Brasil. Río de Janeiro, Brasil: CEPAL.
Herrera, F. (1970). Aspectos institucionales del proceso latinoamericano de integración. En O. Sunkel (Comp.) Integración Política y Económica (pp. 311-344). Santiago de Chile: Editorial Universitaria.

ONU (2011). Base de datos del Comtrade. $<$ http://comtrade. un.org/db/>.

Padilla, C. (2009). La expansión de las industrias extractivas y los desafíos para las organizaciones en América Latina. En CIDSE-ALAI. América Latina: Riqueza privada, pobreza pública. Quito: CIDSE-ALAI, 13-17.

Palma, E. (1999). Actualizar las variables de la integración latinoamericana. En Rolando, F. \& Di Filippo, A. Las dimensiones sociales de la integración regional en América Latina (pp. 151-159). Santiago de Chile: CEPAL.

Prebisch, R. (1959). El Mercado Común Latinoamericano. Comercio Exterior, $I X(5), 25-31$.

Ríos, S. (2003). Mercosur: en busca de una nueva agenda. Mercosur: Dilemas y alternativas de la agenda comercial. (Documento de trabajo, IECI-06c). Buenos Aires, Argentina: BID-INTAL-ITD.

Rosenthal, G. (1999). Procesos de integración regional y las políticas sociales. En Rolando, F. \& Di Filippo, A. Las dimensiones sociales de la integración regional en América Latina (pp. 121-132). Santiago de Chile: CEPAL.

Salazar, M. (2008). Minería-Perú: el precio de la competitividad. $<$ http://ipsnoticias.net/nota.asp?idnews $=87863>$ (Recuperado el 12 de diciembre, 2010).

Sunkel, O. (1998). Desarrollo e integración regional: ¿otra oportunidad para una promesa incumplida? Revista de la CEPAL, Número extraordinario, 229- 241.

Tavares, M., \& Gomes, G. (1998). La CEPAL y la integración económica de América Latina. Revista de la CEPAL, Número extraordinario, 213- 228. 